\title{
Metabolisches Syndrom - Diabetes mellitus Möglichkeiten der Prävention: Was ist sinnvoll und was können wir tun?
}

Stephan Jacob, Frohmut Jacob

Praxis für Prävention und Therapie, Kardio-Metabolisches Institut, Villingen-Schwenningen

Neben den rein medizinischen Kosten werden durch Arbeitsausfall und vorzeitige Berentung erhebliche indirekte Kosten entstehen, die in der Zukunft deswegen eine besondere Rolle spielen werden, da zunehmend gerade junge Patienten mit Typ-2-Diabetes diagnostiziert werden. Diese werden aufgrund der längeren Exposition der Risikofaktoren, wie Hypertonie, Hyperlipidämie und Hyperglykämie, vermutlich vermehrt vaskuläre Komplikationen erleiden und somit wahrscheinlich früher aus dem Arbeitsleben ausscheiden. Dies ist eine der großen Herausforderungen für die Zukunft.

\section{Kardiovaskuläres Risiko} schon bei Diagnose des Diabetes erhöht Betrachtet man konventionell die Bedeutung des Diabetes im Vergleich zu den Nichtdiabetikern dann sieht man, dass Diabetiker gegenüber gesunden eine 5-fach erhöhte kardiovaskuläre Ereignisrate aufweisen. In der Nurses Health Study (NHS) wurden alle Teilnehmer bezüglich des Glukosestoffwechsels untersucht, dabei ergab sich ein überraschendes Bild. Wurden als Referenz bezüglich der kardiovaskulären Ereignisse echt Stoffwechselgesunde mit einem relativen Risiko von 1 gesetzt, zeigten Teilnehmerinnen, die eine frisch entdeckte Störung der Glukosetoleranz (IGT) aufwiesen, bereits eine 2,8-fach erhöhte Ereignisrate, neu entdeckte Diabetespatientinnen sogar eine gegenüber den gesunden auf 3,5-fach erhöhte Rate. Die bekannten Diabetikerinnen wiesen die besagte 5-fach erhöhte Rate auf. Auffällig ist und für die Prävention besonders bedeutsam, dass der deutlichste Anstieg des kardiovaskulären Risikos in einem Bereich erfolgte, der landläufig im Alltag gar nicht entdeckt wird, da die Gruppe mit gestörter Glukosetoleranz (IGT) normalerweise nur durch einen extra durchgeführten Glukosetoleranztest entdeckt werden kann. Auffällig ist auch, dass durch die Progression der Hyperglykämie von gestörter Glukosetoleranz (IGT) zum manifesten Diabetes sich das Risiko „nur“ um 1,7-fach er-
Der Typ-2-Diabetes ist weltweit deutlich am Anwachsen, was mittlerweile nicht nur die im Gesundheitssystem Tätigen, sondern sogar die Politik mit Sorge beschäftigt: denn es sind vor allen Dingen die Kosten, die in den nächsten Jahren erhebliche Probleme bereiten werden. Dabei spielen die Ausgaben für die metabolische Kontrolle eine sehr untergeordnete Rolle im Vergleich zu den Kosten für die Behandlung und die Folgen der Gefäßerkrankungen.

höht, somit die stärkste Erhöhung im prä-diabetischen und damit der normalen klinischen Routine nicht sichtbaren Bereich erfolgte.

Das bedeutet für die Praxis, dass wir bezüglich der Prävention vieler kardiovaskulärer Ereignisse zu spät kommen, wenn der Diabetes bereits diagnostiziert ist. Dies zeigen auch die Beobachtungen, dass bei Diagnose ca. 50\% der Menschen mit Typ-2-Diabetes bereits vaskuläre Komplikationen aufweisen, ja dass sogar die Störung der Glukosetoleranz erst im Rahmen eines Herzinfarktes oder Schlaganfall es aufgefallen ist.

\section{Ursachen für den Anstieg \\ des kardiovaskulären Risikos}

Ursachen für diese Entwicklung sind zwar auch genetische Veranlagungen, jedoch zeigt der Blick in die Geschichte, dass erst mit dem Wohlstand das Körpergewicht, vor allen Dingen der „Wohlstandsbauch“, zum Problem wurde. Leider ist zu erwarten, dass sich aufgrund der Übergewichtassoziierten Erkrankungen in Zukunft die Lebenserwartung trotz des medizinischen Fortschritts leicht reduzieren wird.

Deutschland hat in Europa leider eine unrühmliche Spitzenrolle bezüglich der Häufigkeit des Übergewichtes bei Männern und Frauen: der Report der IASO 2007 zeigte auf, dass in dem Bereich Übergewicht und Adipositas, d.h. BMI $>25 \mathrm{~kg} / \mathrm{m}^{2}$, sowohl bei Männern als auch bei Frauen Deutschland die ersten Plätze belegt. Seit langem ist bekannt, dass mit Zunahme des BMI die kardiovaskuläre Mortalität erheblich ansteigt und zwischen Normalgewicht und Adipositas fast fünffach höher ist. Parallel dazu wird 


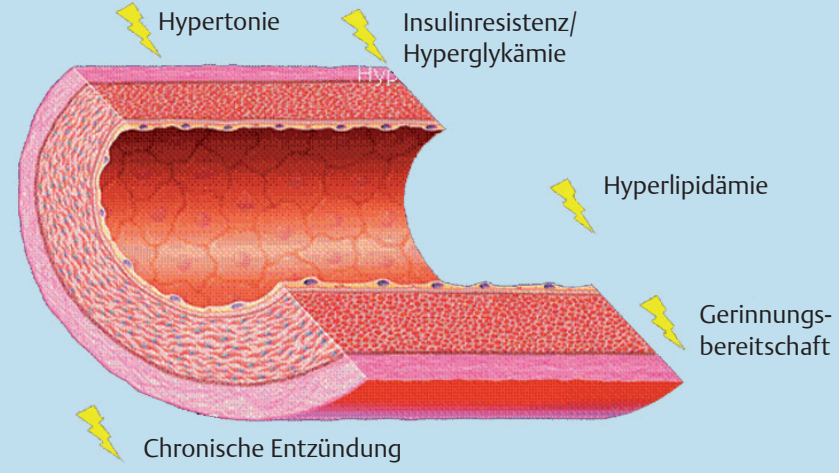

Abb. 1 Wichtige Noxen für die Gefäße bzw. das Endothel bei dem Patienten mit Diabetes mellitus Typ 2.

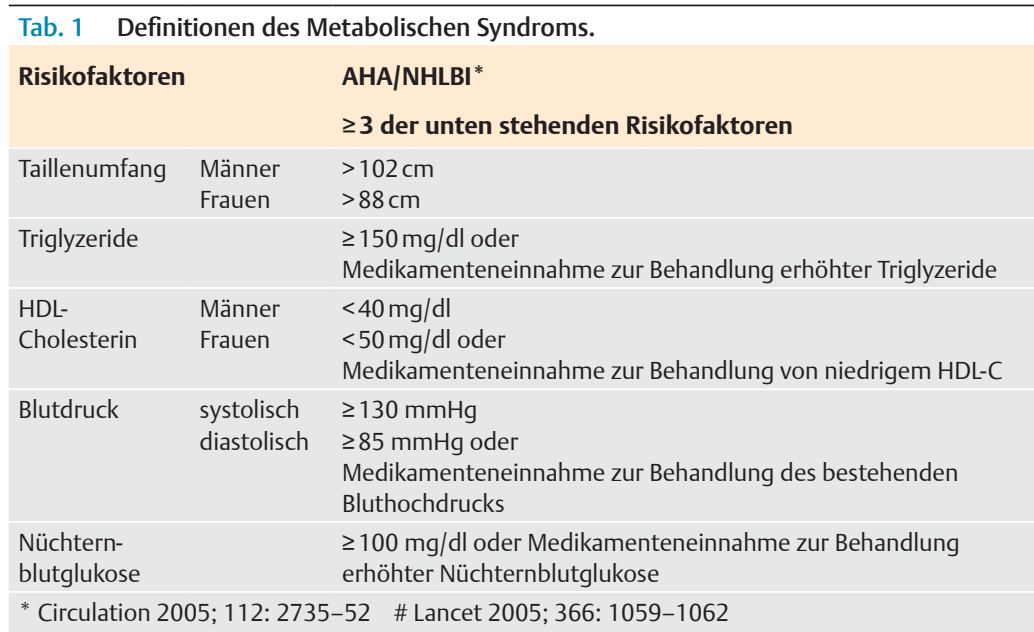

nicht nur eine Erhöhung der Sterblichkeit, sondern auch der Erkrankungshäufigkeit für Brustkrebs, Darmkrebs, Prostatakrebs, Endometrium-, Leber- und Gallenblasenkrebs gesehen.

Die Studie Procam aus Deutschland hat schon 1992 gezeigt, dass mit Zunahme des BMI bestimmte kardiovaskuläre Risikofaktoren gleichzeitig zunehmen. Dabei ist jeder dieser Risikofaktoren einzeln betrachtet, ein anerkannter wichtiger kardiovaskulärer Risikofaktor. So haben Menschen mit einem BMI über $30 \mathrm{~kg} / \mathrm{m}^{2}$ erheblich häufiger erhöhte Werte für Blutdruck, Cholesterin, Triglyzeride, Nüchtern-Blutzucker und erheblich erniedrigtes HDL-Cholesterin. Alles Faktoren, die letztendlich das gleiche Organ schädigen, nämlich das Gefäß, das Endothel.

Tragischerweise treten beim Patienten mit Diabetes mellitus oder dem metabolischen Syndrom gleich mehrere dieser, oft mit dem Übergewicht assoziierten, kardiovaskulären Risikofaktoren gleichzeitig auf (Abb. 1).

Während früher aufgrund der isolierten Sicht der kardiovaskulären Risikofaktoren häufig der Mensch mit Diabetes und leicht erhöhtem Blutdruck, oder auch leicht erhöhten Fettwerten eher abwartend betreut wurde („So hoch ist der Wert nicht, dass wir gleich behandeln müssen“), sehen wir heute beim gleichzeitigen Auftreten dieser Risikofaktoren, auch wenn sie nicht extrem erhöht sind, eine große Gefahr für Herz und Gefäße. Seit der skandinavischen Studie von Isomaa et al. 2001, in der 3500 Menschen über 7 Jahre bezüglich ihrer Mortalität beobachtet wurden, hat sich unsere Sicht geändert: im Vergleich zu Menschen ohne metabolisches Syndrom zeigten die Personen, die das Muster an Risikofaktoren des metabolischen Syndroms zeigten, eine erheblich erhöhte Gesamt- und kardiovaskuläre Mortalität. Bedeutsam ist, dass über $80 \%$ unserer Patienten mit Typ-2-Diabetes dieses metabolische Syndrom aufweisen.

Das Risikomuster des metabolischen Syndroms ist jedoch auch ein Marker für ein erheblich erhöhtes Risiko, einen Typ-2-Diabetes zu entwickeln: in der WOSCOP-Studie fand sich bei fast 6000 ursprünglich nicht-diabetischen Männern eine fast vierfach erhöhte Inzidenz an Typ-2-Diabetes, wenn das metabolische Syndrom vorlag. Das Risiko war 25-fach erhöht, wenn mehr als 4 Punkte des metabolischen Syndroms vorlagen (Tab. 1).

\section{Metabolisches Syndrom nicht nur bei Diabetes relevant}

Betrachtet man Nicht-Diabetiker mit Hypertonie, so zeigt sich, dass auch hier das gleichzeitige Vorliegen weiterer vaskulärer Risikofaktoren des metabolischen Syndroms das Risiko für Organschäden wie linksventrikuläre Hypertrophie, Retinopathie und Mikroalbuminurie mehr als verdoppelt.

Schillaci et al. beschrieben, dass gegenüber Hochdruck-Patienten ohne zusätzliche Risikofaktoren das 10 Jahre ereignisfreie Überleben bei Menschen mit Hypertonie mit zusätzlichen Risikomustern ein signifikant schlechteres Überleben haben.

\section{Situation in Baden-Württemberg}

Für Deutschland gibt es relativ wenig Zahlen. Im Jahre 2004 untersuchten wir in Sankt Georgen nicht-diabetische Personen bezüglich ihres kardiovaskulären Risikos. Über Zeitungen, Flugblätter und Hausärzte wurden gesunde Nicht-Diabetiker angesprochen, und fast 700 Personen wurden untersucht. Diese waren zwischen 18 und 75 Jahre alt, außerdem waren circa 100 Personen pro Altersdekade und je 50\% Männer und Frauen unter den Teilnehmern.

Bezüglich des Gewichts gab es die erste Überraschung: bei diesen - sicherlich präselektionierten - Personen hatten nur 39\% ein normales Gewicht, $41 \%$ wiesen ein Übergewicht auf, während jeder fünfte eine Adipositas hatte. Bedenkt man, dass Diabetiker ausgeschlossen waren, und dass wohl eher nur die Gesundheitsbewussten sich an der Aktion beteiligten, so muss davon ausgegangen werden, dass die Zahl der Übergewichtigen und Adipösen in der Region deutlich höher ist. 
Betrachtet man nun die Häufigkeit des metabolischen Syndroms in dieser Gruppe gemäß der BMI-Klassen, so ergibt sich eine mit dem zunehmenden Übergewicht stark ansteigende Häufigkeit des metabolischen Syndroms, es stieg von 7 bei Normalgewicht auf $31 \%$ bei Übergewicht, bei den Adipösen hatten 2 Drittel der Personen das metabolische Syndrom (Abb. 2).

\section{Metabolisches Syndrom bei bereits}

bestehendem Typ-2-Diabetes häufig

Parallel untersuchten wir 2004 in den Praxen die Häufigkeit des metabolischen Syndroms bei in der Praxis behandelten bekannten Diabetikerinnen und fanden dort die Häufigkeit von über $80 \%$, welches sich mit den Zahlen der internationalen Literatur deckt.

Die mithilfe des Ultraschalls gemessene Intima Media Dicke (IMT) war bei Personen mit dem Risikomuster des metabolischen Syndroms bereits erheblich verdickt, was die erhöhte kardiovaskuläre Gefährdung noch einmal unterstreicht.

\section{Metabolisches Syndrom bereits sehr häufig} bei den Jüngeren!

Bei der Analyse der unterschiedlichen Altersgruppen findet sich bei Menschen über 50 Jahren das metabolische Syndrom bei knapp 40\% (ohne Geschlechtsunterschied). Überraschend zeigte sich bereits in der Gruppe der 20-40-Jährigen bei Männern mit über 20\% das metabolische Syndrom, während es bei Frauen unter 10\% lag. In der Gruppe der 40-50-Jährigen jedoch, hatten auch die Frauen „aufgeholt“: die Häufigkeit lag auch hier bei knapp 20\%. Somit ergibt sich ein doch erheblicher Handlungsbedarf besonders bei Männern in der Altersgruppe der 20-40-Jährigen, denn dort wurde bei jedem fünften Mann bereits ein metabolisches Syndrom gesehen (Abb. 3).

Das Auftreten der metabolischen Risikofaktoren bei den jungen Generationen lässt leider befürchten, dass wir in der Zukunft eine erhebliche Zunahme an kardiometabolischen Erkrankungen sehen werden, insbesondere da durch das gleichzeitige Vorhandensein mehrerer Risikofaktoren das Risiko potenziert wird. Daher ist es höchste Zeit etwas zu unternehmen (Info 1)!

\section{Pråvention ist dringend erforderlich}

Da die wichtigsten Risikofaktoren für die Entwicklung des Typ-2-Diabetes gut bekannt sind, wurde auch überprüft, wie effektiv die frühzeitige Intervention ist: in der DPP und DPS wurde eindrücklich gezeigt, dass durch eine intensive Lebensstilintervention das Risiko für die Entwicklung eines Typ-2-Diabetes bei Menschen mit bereits bestehender Glukosetoleranz (IGT) erheblich reduziert werden kann:

- während das Neu-Auftreten des Diabetes in der Kontrollgruppe fast $36 \%$ in 4 Jahren betrug, konnte dieses Risiko durch intensive Lebensstiländerungen praktisch halbiert werden.

- NNT Es müssen nurca. 10 Patienten behandelt werden, um einen neuen Diabetiker zu verhindern!

\section{Auch Pharmakotherapie erfolgreich}

Bestimmte Medikamente haben gezeigt, dass sie das Auftreten von Typ-2-Diabetes verhindern, andere sind mit einem vermehrten Auftreten von Typ-2-Diabetes assoziiert (Tab. 2).

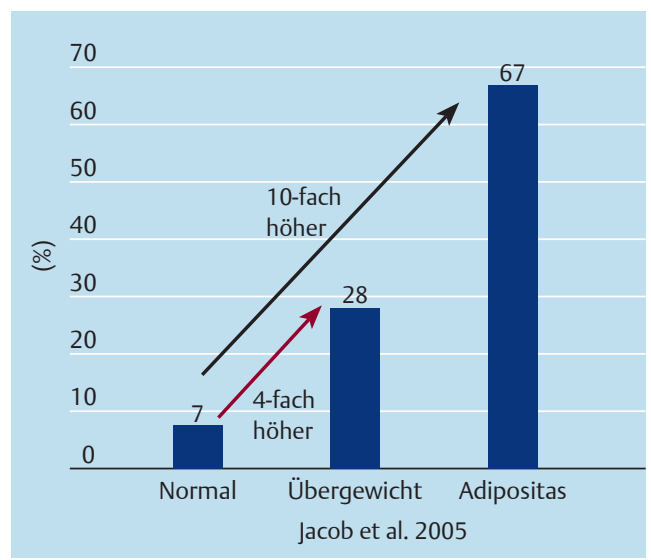

Abb. 2 Häufigkeit des metabolischen Syndroms bei Nicht-Diabetikern - BMI.
Info 1

Wohlstanderkrankungen sind auf dem Vormarsch!

Das metabolische Syndrom ist:

- assoziiert mit erhöhtem Risiko für kardiovaskuläre Probleme (auch ohne Vorliegen eines Diabetes)

- assoziiert mit

erhöhtem Risiko für Typ-2Diabetes

- in Baden-Württemberg bei Erwachsenen sehr häufig

- volksgesundheitlich und -wirtschaftlich sehr relevant!

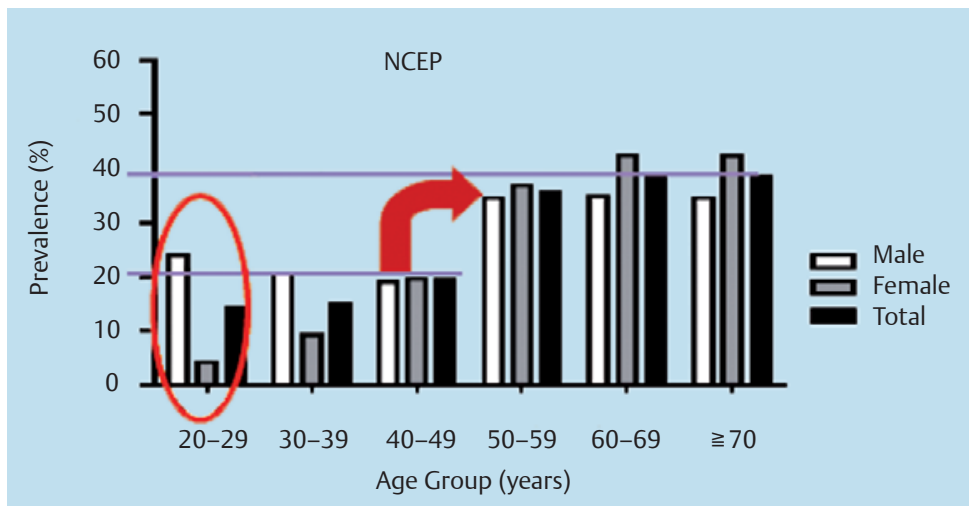

Omari A, Winkler K, Jacob F, Balletshofer B, Wahl HG, Keulen L, Caterson ID, März W, Probst J, Jacob S

Abb. 3 Metabolisches Syndrom, Alter und Geschlecht.

\begin{tabular}{|c|c|}
\hline \multicolumn{2}{|c|}{$\begin{array}{l}\text { Tab. } 2 \text { Wirkung von Medikamenten auf Typ-2- } \\
\text { Diabetes. }\end{array}$} \\
\hline $\begin{array}{l}\text { Intervention reduziert } \\
\text { Neuauftreten } \\
\text { von Typ-2-Diabetes }\end{array}$ & $\begin{array}{l}\text { Vermehrtes Auftreten von } \\
\text { Typ-2-Diabetes }\end{array}$ \\
\hline Lebensstil-Änderung & Atenolol \\
\hline Metformin & Metoprolol \\
\hline Orlistat & HCT (hochdosiert) \\
\hline \multicolumn{2}{|l|}{ Acarbose } \\
\hline \multicolumn{2}{|l|}{ Pioglitazon } \\
\hline $\begin{array}{l}\text { AT1-Blocker } \\
\text { (z. B. Valsartan = Navigator- } \\
\text { Studie) }\end{array}$ & \\
\hline ACE-Hemmer & \\
\hline
\end{tabular}


Tab. 3 Metabolic clinic Sydney.

\begin{tabular}{|c|c|c|c|c|}
\hline & \multicolumn{2}{|l|}{ Mean (Range) } & \multirow{2}{*}{$\begin{array}{l}\text { Change } \\
\text { (\%) }\end{array}$} & \multirow[t]{2}{*}{$\mathbf{p}$} \\
\hline & Baseline & 12 months & & \\
\hline Weight $(\mathrm{kg})$ & $96,4(75.5-137.8)$ & $86.9(63.7-133.7)$ & -9.8 & $<0.001$ \\
\hline Waist circumference $(\mathrm{cm})$ & $112.0(89-140)$ & $99.0(76-128.5)$ & -11.1 & $<0.001$ \\
\hline Glycated Haemoglobin (\%) & $8.1(5.8-12.4)$ & $6.9(5.3-9.2)$ & -14.6 & $<0.001$ \\
\hline Systolic Blood Pressure (mmHg) & $141(120-180)$ & $125(110-150)$ & -11.2 & $<0.001$ \\
\hline Diastolic Blood Pressure $(\mathrm{mmHg})$ & $80.5(65-95)$ & $73(65-85)$ & -10.0 & 0.001 \\
\hline HDL Cholesterol (mmol/L) & $1.2(0.8-1.9)$ & $1.5(0.8-2.1)$ & +14.0 & 0.01 \\
\hline Triglycerides (mmol/L) & $1.9(0.5-4.1)$ & $1.5(0.6-3.0)$ & -24.0 & 0.001 \\
\hline
\end{tabular}

Viele Interventionsstudien unterstreichen die Wertigkeit einer intensiven Lebensstilintervention. Interessant ist eine Beobachtung aus der TULIP, in der Menschen mit erhöhtem metabolischen Risiko eine intensive Lebensstiländerung anstrebten und mit aufwendigen Forschungsmethoden untersucht wurden. Dabei ergab sich ein überraschender Befund: bei einer Zwischenanalyse zeigten die Teilnehmer nach circa 9 Monaten lediglich eine sehr moderate Gewichtsreduktion von $2,4 \mathrm{~kg}$, das entsprach einer Reduktion des Körpergewichtes um 3\% und erscheint wenig effektiv. Bei der genauen Untersuchung zeigte sich jedoch, dass das viszerale Fett überproportional stark abgenommen hatte, verbunden mit einer deutlichen Verbesserung der Insulinwirkung, einer Reduktion des Blutzucker, der Blutfette und der Blutdruckwerte sowie einer erheblichen Reduktion der Entzündungswerte. Weiterhin zeigten sich eine signifikante Verbesserung der Gefäßdicke und der Endothelfunktion. Ein Ergebnis, das niemand beim Blick auf die Waage erwartet hätte. Untersuchungen aus Australien zeigen, dass auch bei adipösen Diabetikern ein intensives Lebensstil-Coaching sehr erfolgreich sein kann. In einem interdisziplinären Team von BewegungsTherapeuten, Psychologen, Ernährungsberatern und Ärzten gelang es den Patienten, nach einem Jahr circa $10 \mathrm{~kg}$ Gewicht zu reduzieren, den Bauchumfang zu reduzieren sowie die Einstellung der kardio-metabolischen Risikofaktoren erheblich zu verbessern (Tab. 3).

Das Besondere bei dieser Studie ist, dass bereits nach circa 3 Monaten des Programms bei den meisten Patienten die medikamentöse Therapie adaptiert werden musste und reduziert werden konnte, sodass eine deutliche Einsparung bezüglich der Kosten für Medikamente erreicht wurde. Damit könnte dieses Programm sich fast selbst tragen, eine große Studie in USA, die LOOK AHEAD Studie bestätigte diese Beobachtungen in einer sehr großen Population.

Prävention ist machbar - auch evidenzbasiert - wir könnten handeln

Wenn nun die Datenlage derart klar ist, so muss man sich fragen, warum wir immer noch diese Probleme haben.
Ein Grund dafür ist, dass derzeit relativ wenige Angebote zur intensiven Lebensstil-Intervention und Coaching wohnortnah angeboten werden. Viele der Aktionen sind unkoordiniert, kurzfristig angelegt und leider oft auch nicht qualitätskontrolliert und meist auf das Engagement einzelner angewiesen. Außerdem gibt es kaum ausgebildete Trainer und Therapeuten für unsere Patienten (z. B. gibt es immer noch keine gut ausgebildeten Bewegungstherapeuten für Kranke). Weiterhin sind bei vielen dieser „Insellösungen“ die Ärzte oft überhaupt nicht eingebunden: Präventionsangebote werden aber auch von den Kostenträgern durchaus leider eingesetzt, um für das Leistungsangebot ihrer Kasse zu werben (Nur gut fürs Marketing).

\section{Besteht Bedarf?}

In einer wissenschaftlichen Untersuchung haben wir Patienten in Arztpraxen befragt zu den bisherigen Angeboten bezüglich LebensstilCoaching. Dabei ergab sich, dass sehr viele Patienten bisher nur kurzfristig und begrenzt, wenn überhaupt, Hilfe angeboten bekamen, am häufigsten Ernährungsberatung bei der Kasse. Bewegungsangebote, vor allen Dingen koordinierter Art, fehlten meist. Dennoch wären fast alle Patienten bereit, mindestens 1-2-mal pro Woche zu einem Zentrum zu gehen, welches diese Lebensstil-Betreuung anbietet. Das Interesse ist vor allem dann groß, wenn diese Maßnahmen auch von den Kostenträgern mit unterstützt werden.

\section{Diabetes kommt nicht über Nacht}

Typ-2-Diabetes und auch das metabolische Syndrom sind typische Wohlstandserkrankungen, die sich nicht über Nacht entwickeln und oft eine lange „Vorlaufstrecke“ haben. Die Umsetzung von Lebensstiländerungen ist daher auch nicht rein kognitiv vermittelbar und umsetzbar. Gewohnheiten, die sich über Jahre entwickelt haben, können auch nicht über Nacht geändert werden und brauchen einen langfristigen Ansatz. Daher ist eine langfristige empathische Betreuung erforderlich. Die Experten müssen die verschiedenen Interventionen koordinieren und gegebenenfalls modifizieren. Dazu gehört auch gegebenenfalls eine begleitende Pharmakotherapie.

\section{Prävention ist nicht nur Früherkennung!}

Es ist fragwürdig, wenn durch frühe Erkennungsmaßnahmen zwar Patienten mit erhöhtem Risiko identifiziert werden, dadurch bei vielen Angst und Sorge vor einer möglichen Erkrankung hervorgerufen, aber dann keine nachhaltige Betreuung angeboten wird. Die Risikopersonen brauchen Unterstützung und werden oft mit allgemeinen Ratschlägen alleine gelassen (Abb. 4). 
Mit „FDH“ und „bewege dich mehr“ kann das Problem nicht gelöst werden. Die Angebote in Funk, Presse, TV und Internet sind oft rein kommerziell und sehr häufig medizinisch extrem fragwürdig. Dies kann aber der Laie häufig nicht erkennen. Daher brauchen die Patienten qualifizierte Betreuung (Info 2).

\section{Fazit}

Wir stecken in einem Dilemma: Es wird überall erkannt, dass die Wohlstandserkrankungen erheblich zunehmen und enorme Ressourcen benötigen. Interventionsstudien, die durch Forschungsmittel gut ausgestattet waren, beweisen, dass Intervention möglich und Prävention sinnvoll ist. Die Umsetzung im Alltag ist jedoch erheblich schwieriger, schon allein daher, weil entsprechende Strukturen fehlen aber auch insbesondere, weil die Koordination und kompetente Überwachung/Steuerung dieser Präventionsleistungen derzeit kaum erfolgt, die Angebote nicht transparent sind und eine langfristige Finanzierung nicht gesichert ist. Präventionsmaßnahmen werden leider auch zumindest teilweise für Marketingzwecke eingesetzt.

Wir brauchen unbedingt neue Strukturen und multidisziplinäre Ansätze, die nachhaltig realisierbar und finanzierbar sind. Und wir brauchen auch politische Voraussetzungen: Wir haben Institutionen mit Experten und aufwendigen Geräten zur Unterstützung und „Reparatur“ bei Krankheit, dies sind die Krankenhäuser. Wir haben aber keine Institutionen mit Experten, die langfristig, präventiv Gesunderhaltung unterstützen. Derzeit wird überall erkannt, dass die Wohlstandserkrankungen erheblich zunehmen und enorme Ressourcen benötigen. Über Prävention zu reden ist „schick“ (Marketing...)! Doch echt was zu unternehmen ist in Deutschland unter den derzeitigen Strukturen kaum möglich weder für Arzt, noch für Patient, noch für den, der gesund bleiben will. Wir brauchen hierfür unbedingt neue Strukturen und nachhaltige Ansätze, die auch realisier- und finanzierbar sind.

\section{Literatur}

1 Després J-P, Lemieux I, Prud'homme D. Treatment of obesity: need to focus on high risk abdominally obese patients. BMJ 2001; 322: 716-720

2 Eckel RH, Grundy SM, Zimmet PZ. The metabolic syndrome. Lancet 2005; 365: 1415-1428

3 Isomaa B, Almgren P, Tuomi T et al. Cardiovascular morbidity and mortality associated with the metabolic syndrome. Diabetes Care 2001; 24: 683-689

4 Jacob S, Halle M. Verbesserung der kardiometabolischen Prognose- Was können Typ-2-Diabetiker mit einer Lebensstiländerung erreichen? Cardiovasc 2006; 5: 30-33

5 Knowler WC, Barrett-Connor E, Fowler SE et al. Diabetes Prevention Program Research Group. Reduction in the incidence of type 2 diabetes with lifestyle intervention or metformin. $\mathrm{N}$ Engl J Med 2002; 346: 393-403

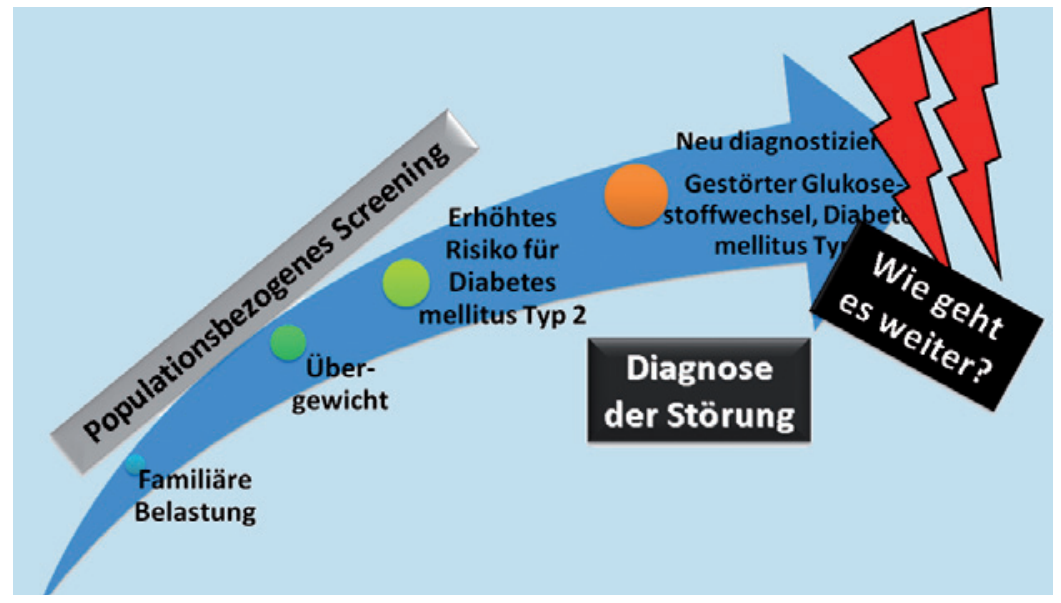

6 Lindstrom J, Ilanne-Parikka P, Peltonen $\mathrm{M}$ et al. Finnish Diabetes Prevention Study Group. Sustained reduction in the incidence of type 2 diabetes by lifestyle intervention: follow-up of the Finnish Diabetes Prevention Study. Lancet 2006; 368: 1673-1679

7 Orchard T], Temprosa M, Goldberg R et al. Diabetes Prevention Program Research Group. The effect of metformin and intensive lifestyle intervention on the metabolic syndrome: the Diabetes Prevention Program randomized trial. Ann Intern Med 2005; 142: 611-619

8 Schaper F, Ott P, Hanefeld M. Adipositas, Bluthochdruck, Dyslipidämie und Dysglykämie Multimodales Management gegen das metabolische Syndrom. Klinikarzt 2005; 34: 359-364

9 Jacob S. Das Kardio-Metabolische Syndrom. Diabetes aktuell 2009; 4: 46-52

10 Tuomilehto J, Lindström J, Eriksson JG et al. Prevention of type 2 diabetes mellitus by changes in lifestyle among subjects with impaired glucose tolerance. N Engl J Med 2001; 344: 1343-1350

11 Gæde P, Vedel P, Larsen N et al. Multifactorial Intervention and Cardiovascular Disease in Patients with Type 2 Diabetes. N Engl J Med 2003; 348: 383-393

12 Omari A, Winkler K, Jacob F et al. Metabolic Syndrome, sex and age. Presented at the Australian Diabetes meeting 2005

13 Pi-Sunyer X, Blackburn G, Brancati FL et al. Reduction in weight and cardiovascular disease risk factors in individuals with type 2 diabetes: one-year results of the look AHEAD trial. Diabetes Care 2007; 30: 1374-1383

14 Wadden TA, Neiberg RH, Wing RR et al. Four-Year Weight Losses in the Look AHEAD Study: Factors Associated With Long-Term Success. Obesity (Silver Spring) 2011; 19: 1987-1998

15 Wen CP, Wai JP, Tsai MK et al. Minimum amount of physical activity for reduced mortality and extended life expectancy: a prospective cohort study. Lancet 2011; 378: 1244-1253
Abb. 4 Entdeckung von Personen mit erhöhtem Risiko durch populationsbasierte Screeningmaßnahmen sollten die Intervention mit anbieten, denn sonst sind die Betroffenen häufig alleine gelassen.

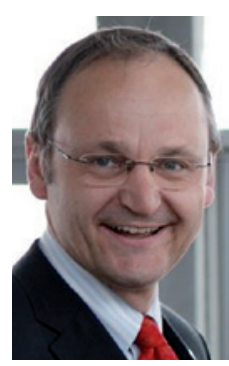

Korrespondenz

Prof. Dr. med. Stephan Jacob Villingen-Schwenningen

\section{Autorenerklärung}

Die Autoren erklären, dass für diesen Artikel

keine Interessenkonflikte bestehen.
Metabolic Syndrome - Diabetes Mellitus

Possibilities for Prevention: What is Useful and What can We Do?

Type 2 diabetes is clearly increasing worldwide and in the meantime is not just a problem for the health-care systems but is also being followed with concern by the political apparatus: it is above all the costs that will cause appreciable problems in the coming years. Here the costs for metabolic check-ups play a very minor part in comparison to the costs for the treatment and sequelae of the associated vascular diseases.

\section{Key words}

metabolic syndrome - diabetes mellitus - health-care systems - costs 